\title{
AVOIDING CONJUGACY CLASSES ON THE 5-LETTER ALPHABET
}

\author{
Golnaz BADKOBEH* AND PASCAL OCHEM ${ }^{\dagger, \ddagger}$
}

\begin{abstract}
We construct an infinite word $w$ over the 5-letter alphabet such that for every factor $f$ of $w$ of length at least two, there exists a cyclic permutation of $f$ that is not a factor of $w$. In other words, $w$ does not contain a non-trivial conjugacy class. This proves the conjecture in Gamard et al. [Theoret. Comput. Sci. 726 (2018) 1-4].
\end{abstract}

Mathematics Subject Classification. 68R15.

Received November 14, 2018. Accepted February 18, 2020.

\section{INTRODUCTION}

A pattern $p$ is a non-empty finite word over an alphabet $\Delta=\{A, B, C, \ldots\}$ of capital letters called variables. An occurrence of $p$ in a word $w$ is a non-erasing morphism $h: \Delta^{*} \rightarrow \Sigma^{*}$ such that $h(p)$ is a factor of $w$. The avoidability index $\lambda(p)$ of a pattern $p$ is the size of the smallest alphabet $\Sigma$ such that there exists an infinite word over $\Sigma$ containing no occurrence of $p$. Bean et al. [2] and Zimin [8] characterized unavoidable patterns, i.e., such that $\lambda(p)=\infty$. However, determining the exact avoidability index of an avoidable pattern requires more work. Although patterns with index 4 [2] and 5 [4] have been found, the existence of an avoidable pattern with index at least 6 is an open problem since 2001.

Some techniques in pattern avoidance start by showing that the considered word avoids other structures, such as generalized repetitions $[6,7]$. Let us say that a word has property $P_{i}$ if it does not contain all the conjugates of the same word $w$ with $|w| \geqslant i$. Recently, in order to study the avoidance of a kind of patterns called circular formulas, Gamard et al. [5] obtained that there exists

- a morphic binary word satisfying $P_{5}$,

- a morphic ternary word satisfying $P_{3}$,

- a morphic word over the 6-letter alphabet satisfying $P_{2}$.

Recall that a pure morphic word is of the form $m^{\omega}(0)$ and a morphic word is of the form $h\left(m^{\omega}(0)\right)$ for some morphisms $m$ and $h$. Independently, Bell and Madill [3] obtained a pure morphic word over the 12-letter alphabet that also satisfies $P_{2}$ and some other properties.

\footnotetext{
Keywords and phrases: Combinatorics on words, conjugacy classes.

1 Goldsmiths, University of London.

2 LIRMM, Université de Montpellier, CNRS, Montpellier, France.

* Golnaz Badkobeh is supported by the Leverhulme Trust on the Leverhulme Early Career Scheme.

$\dagger$ This work is supported by the ANR project CoCoGro (ANR-16-CE40-0005).

$\ddagger$ Corresponding author: ochem@lirmm.fr
} 
It is conjectured that the smallest alphabet allowing an infinite word satisfying $P_{2}$ has 5 letters [5], which is best possible. In this paper, we prove this conjecture using a morphic word. This settles the topic of the smallest alphabet needed to satisfy $P_{i}$.

\section{MAIN RESUlT}

Let $\varepsilon$ denote the empty word. We consider the morphic word $w_{5}=G\left(F^{\omega}(0)\right)$ defined by the following morphisms.

$$
\begin{array}{ll}
F(0)=01, & G(0)=\text { abcd } \\
F(1)=2, & G(1)=\varepsilon, \\
F(2)=03, & G(2)=\text { eacd } \\
F(3)=24, & G(3)=\text { becd } \\
F(4)=23 . & G(4)=\text { be }
\end{array}
$$

Theorem 2.1. The morphic word $w_{5} \in \Sigma_{5}^{*}$ avoids every conjugacy class of length at least 2.

In order to prove this theorem, it is convenient to express $w_{5}$ with the larger morphisms $f=F^{3}$ and $g=G \circ F^{2}$ given below. Clearly, $w_{5}=g\left(f^{\omega}(0)\right)$.

$$
\begin{array}{ll}
f(0)=01203, & g(0)=\text { abcdeacd } \\
f(1)=0124, & g(1)=\text { abcdbecd } \\
f(2)=0120323, & g(2)=\text { abcdeacdbe } \\
f(3)=01240324, & g(3)=\text { abcdbecdeacdbecd, } \\
f(4)=01240323 . & g(4)=\text { abcdbecdeacdbe }
\end{array}
$$

\subsection{Avoiding conjugacy classes in $F^{\omega}(0)$}

Here we study the pure morphic word and the conjugacy classes it contains.

Lemma 2.2. The infinite word $F^{\omega}(0)$ contains only the conjugacy classes listed in $C=$ $\left\{F(2), F^{2}(2), F^{d}(4), f^{d}(0)\right\}$, for all $d \geqslant 1$.

Proof. Notice that the factor 01 only occurs as the prefix of the $f$-image of every letter in $F^{\omega}(0)$. Moreover, every letter 1 only occurs in $F^{\omega}(0)$ as the suffix of the factor 01 . Let us say that the index of a conjugacy class is the number of occurrences of 1 in any of its elements. An easy computation shows that the set of complete conjugacy classes in $F^{\omega}(0)$ with index at most one is $C_{1}=\left\{F(2), F^{2}(2), F(4), F^{2}(4), f(4), f(0)\right\}$. Let us assume that $F^{\omega}(0)$ contains a conjugacy class $c$ with index at least two. Let $w \in c$ be such that 01 is a prefix of $w$. We write $w=p s$ such that the leftmost occurrence of 01 in $w$ is the prefix of $s$. Then the conjugate $s p$ of $w$ also belongs to $c$ and thus is a factor of $F^{\omega}(0)$. This implies that the pre-image $v=f^{-1}(w)$ is a factor of $F^{\omega}(0)$, and so does every conjugate of $v$. Thus, $F^{\omega}(0)$ contains a conjugacy class $c^{\prime}$ such that the elements of $c$ with prefix 01 are the $f$-images of the elements of $c^{\prime}$. Moreover, the index of $c^{\prime}$ is strictly smaller than the index of $c$.

Using this argument recursively, we conclude that every complete conjugacy class in $F^{\omega}(0)$ has a member of the form $f^{i}(x)$ such that $x$ is an element of a conjugacy class in $C_{1}$.

Now we show that $F(2)$ does not generate larger conjugacy classes in $F^{\omega}(0)$. We thus have to exhibit a conjugate of $f(F(2))=F^{4}(2)=0120301240324$ that is not a factor of $F^{\omega}(0)$. A computer check shows that the conjugate 4012030124032 is not a factor of $F^{\omega}(0)$. Similarly, $F^{2}(2)$ does not generate larger conjugacy classes in $F^{\omega}(0)$ since the conjugate 301203012401203230124032 of $f\left(F^{2}(2)\right)=F^{5}(2)=012030124012032301240323$ is not a factor of $F^{\omega}(0)$. 


\subsection{Avoiding conjugacy classes in $w_{5}$}

We are ready to prove Theorem 2.1. A computer $\operatorname{check}^{1}$ shows that $w_{5}$ avoids every conjugacy class of length at most 1000. Let us assume that $w_{5}$ contains a conjugacy class $c$ of length at least 41. Consider a word $w \in c$ with prefix ab. Notice that ab only appears in $w_{5}$ as the prefix of the $g$-image of every letter. Since $|w| \geqslant 41$, $w$ contains at least 2 occurrences of $\mathrm{ab}$ and we write $w=p s$ such that the rightmost occurrence of ab in $w$ is the prefix of $s$. Then the conjugate $s p$ of $w$ also belongs to $c$ and thus is a factor of $w_{5}$. This implies that the pre-image $v=g^{-1}(w)$ is a factor of $F^{\omega}(0)$, and so does every conjugate of $v$. Thus, $F^{\omega}(0)$ contains a conjugacy class $c^{\prime}$ such that the elements of $c$ with prefix ab are the $f$-images of the elements of $c^{\prime}$.

To finish the proof, it is thus sufficient to show that for every $c^{\prime} \in C$, there exists a conjugate of $g\left(c^{\prime}\right)$ that is not a factor of $w_{5}$. Recall that $C=\left\{F(2), F^{2}(2), F^{d}(4), f^{d}(0)\right\}$ for all $d \geqslant 1$. The computer check mentioned above settles the case of $F(2)$ and $F^{2}(2)$ since $|g(F(2))|<\left|g\left(F^{2}(2)\right)\right|=40<1000$. It also settles the case of $f(4)$ and $f(0)$ since $|g(f(0))|<|g(f(4))|=90<1000$.

The next four lemmas handle the remaining cases (with $d \geqslant 1$ ):

$-g\left(f^{d}(F(4))\right)=g\left(f^{d}(23)\right)$

$-g\left(f^{d}\left(F^{2}(4)\right)\right)=g\left(f^{d}(0324)\right)$

$-g\left(f^{d+1}(4)\right)=g\left(f^{d}(01240323)\right)$

$-g\left(f^{d+1}(0)\right)=g\left(f^{d}(01203)\right)$

Notice that for technical reasons, we do not consider $g(f(4))$ and $g(f(4))$, which are also covered by the computer check.

Lemma 2.3. Let $p_{23}=e . g\left(3 f(3) \ldots f^{d-1}(3) \cdot f^{d}(3)\right) \quad$ and $\quad s_{23}=g\left(f^{d-1}(01203) \cdot f^{d-2}(01203) \ldots\right.$ $f(01203) 01203) . a b c d e a c d b$. For every $d \geqslant 0$, the word $T_{23}=p_{23} s_{23}$ is a conjugate of $g\left(f^{d}(23)\right)$ that is not a factor of $w_{5}$.

Proof. It is easy to check that $T_{23}$ is indeed a conjugate of $g\left(f^{d}(23)\right)$. Let us assume that $T_{23}$ appears in $w_{5}$.

The letter 3 in $f^{\omega}(0)$ appears after either 0 or 2. However e is a suffix of $g(2)$ and not of $g(0)$. Therefore, e. $g(3)$ is a suffix of $g(23)$ only. Since 23 is a suffix of $f(2)$ and not of $f(0)$, then $g(23 f(3))$ is a suffix of $g(f(23))$ only. Using this argument recursively, $p_{23}$ is a suffix of $g\left(f^{d}(23)\right)$ only.

Now, the letter 3 in $f^{\omega}(0)$ appears before either 0 or 2, however abcdeacdb is a prefix of $g(2)$ and not of $g(0)$. Thus $g(01203)$.abcdeacdb is a prefix of $g(012032)$ only. Since 012032 is a prefix of $f(2)$ and not of $f(0)$, then $g(f(01203) 012032)$ is a prefix of $g(f(012032))$ only. Using this argument recursively, $s_{23}$ is a prefix of $g\left(f^{d-1}(012032)\right)$ only. Thus, if $T_{23}$ is a factor of $w_{5}$, then $g\left(f^{d}(232)\right)$ is a factor of $w_{5}$. This is a contradiction since 232 is not a factor of $f^{\omega}(0)$.

Lemma 2.4. Let $p_{0324}=$ acdbecd.g $\left.\left(24 f(24) \ldots f^{d-1}(24)\right) \cdot f^{d}(24)\right)$ and $s_{0324}=g\left(f^{d-1}(01240) \ldots\right.$ $f(01240) .01240)$.abcdbecde. For every $d \geqslant 0$, the word $T_{0324}=p_{0324} g\left(f^{d}(0)\right) s_{0324}$ is a conjugate of $g\left(f^{d}(0324)\right)$ that is not a factor of $w_{5}$.

Proof. Let us assume that $T_{0324}$ appears in $w_{5}$.

The letter 2 in $f^{\omega}(0)$ appears after either 1 or 3. However acdbecd is a suffix of $g(3)$ and not of $g(1)$. Therefore acdbecd. $g(24)$ is a suffix of $g(324)$ only. Since 324 is a suffix of $f(3)$ and not of $f(1)$, then $g(324 f(24))$ is a suffix of $g(f(324))$ only. Using this argument recursively, $p_{0324}$ is a suffix of $g\left(f^{d}(324)\right)$ only.

Now, the letter 0 in $f^{\omega}(0)$ appears before either 1 or 3 . However abcdbecde is a prefix of $g(3)$ and not of $g(1)$. Thus $g(01240)$.abcdbecde is a prefix of $g(012403)$ only. Since 012403 is a prefix of $f(3)$ and not of $f(1)$, then $g(f(01240) 012403)$ is a prefix of $g(f(012403))$ only. Using this argument recursively, $s_{0324}$ is a prefix of $g\left(f^{d-1}(012403)\right)$ only. Thus, if $T_{0324}$ is a factor of $w_{5}$, then $g\left(f^{d}(32403)\right)$ is a factor of $w_{5}$. This is a contradiction since 32403 is not a factor of $f^{\omega}(0)$.

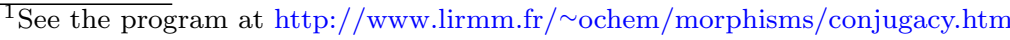


Lemma 2.5. Let $p_{01240323}=$ ecdeacdbe.g(0323f(0323) $\left.\cdots f^{d-1}(0323) \cdot f^{d}(0323)\right)$ and $s_{01240323}=$ $g\left(f^{d}(012) f^{d-1}(012) . \cdots f(012) 012\right) . a b c d b$. For every $d \geqslant 0$, the word $T_{01240323}=p_{01240323} s_{01240323}$ is a conjugate of $g\left(f^{d}(01240323)\right.$ that is not a factor of $w_{5}$.

Proof. Let us assume that $T_{01240323}$ appears in $w_{5}$.

The factor 03 in $f^{\omega}(0)$ appears after either 2 or 4 . However ecdeacdbe is a suffix of $g(4)$ and not of $g(2)$. Therefore ecdeacdbe. $g(0323)$ is a suffix of $g(40323)$ only. Since 40323 is a suffix of $f(4)$ and not of $f(2)$, then $g(40323 f(0323))$ is a suffix of $g(f(40323))$, using this argument recursively, $p_{01240323}$ is a suffix of $g\left(f^{d}(40323)\right)$ only.

Now, the factor 12 in $f^{\omega}(0)$ appears before either 0 or 4 . However abcdb is a prefix of $g(4)$ and not of $g(0)$. Thus $g(012)$.abcdb must only be a prefix of $g(0124)$ and since 0323 is a prefix of $f(4)$ and not of $f(0)$ then $g(f(012) 0124)$ is a prefix of $g(f(0124))$ only. Using this argument recursively, $s_{01240323}$ is a prefix of $g\left(f^{d}(0124)\right)$ only. Thus, if $T_{01240323}$ is a factor of $w_{5}$, then $g\left(f^{d}(403230124)\right)$ is a factor of $w_{5}$. This is a contradiction since 403230124 is not a factor of $f^{\omega}(0)$.

Lemma 2.6. Let $p_{01203}=\operatorname{d.g}\left(3 f(3) \ldots f^{d-1}(3) . f^{d}(3)\right) \quad$ and $s_{01203}=g\left(f^{d}(012) f^{d-1}(012) . f^{d-2}(012) \ldots\right.$ $f(012) 012)$.abcdeac. For every $d \geqslant 0$, the word $T_{01203}=p_{01203} s_{01203}$ is a conjugate of $g\left(f^{d}(01203)\right)$ that is not a factor of $w_{5}$.

Proof. Let us assume that $T_{01203}$ appears in $w_{5}$.

The letter 3 in $f^{\omega}(0)$ appears after either 0 or 2. however d is a suffix of $g(0)$ and not of $g(2)$. Therefore d.g(2) is a suffix of $g(12)$ only. Since 12 is a suffix of $f(1)$ and not of $f(3)$, then $g(12 f(2))$ is a suffix of $g(f(12))$ only. Using this argument recursively, $p_{01203}$ is a suffix of $g\left(f^{d}(12)\right)$ only.

Now, 012 in $f^{\omega}(0)$ appears before either 1 or 4 , however abcdeac is only a prefix of $g(1)$ and not of $g(4)$. Thus $g$ (012).abcdeac is a prefix of $g(0120)$ only. Since 0120 is a prefix of $f(1)$ and not of $f(4)$, then $g(f(012) 0120)$ is a prefix of $g(f(0120))$ only. Using this argument recursively, $s_{01203}$ is a prefix of $g\left(f^{d}(0120)\right)$. Thus, if $T_{01203}$ is a factor of $w_{5}$, then $g\left(f^{d}(030120)\right)$ is a factor of $w_{5}$. This is a contradiction since 030120 is not a factor of $f^{\omega}(0)$.

\section{REFERENCES}

[1] K.A. Baker, G.F. McNulty and W. Taylor, Growth problems for avoidable words. Theoret. Comput. Sci. 69 (1989) 19-345.

[2] D.R. Bean, A. Ehrenfeucht and G.F. McNulty, Avoidable patterns in strings of symbols. Pacific J. Math. 85 (1979) $261-294$.

[3] J.P. Bell and B.W. Madill, Iterative Algebras. Algebr. Represent. Theor. 18 (2015) 1533-1546.

[4] R.J. Clark, Avoidable formulas in combinatorics on words. Ph.D. thesis, University of California, Los Angeles. Available at http://www.lirmm.fr/ ochem/morphisms/clark_thesis.pdf (2001).

[5] G. Gamard, P. Ochem, G. Richomme and P. Séébold, Avoidability of circular formulas. Theoret. Comput. Sci. 726 (2018) $1-4$.

[6] L. Ilie, P. Ochem and J.O. Shallit, A generalization of repetition threshold. Theoret. Comput. Sci. 92 (2004) 71-76.

[7] P. Ochem, A generator of morphisms for infinite words. RAIRO: ITA 40 (2006) 427-441.

[8] A.I. Zimin, Blocking sets of terms. Math. USSR Sbornik 47 (1984) 353-364. 